\title{
DETERMINATION OF THE BACTERIAL COMPOSITION OF A FULL-SCALE MULTI-LAYER BIOFILTER SYSTEM BASED ON 454 PYROSEQUENCING
}

\author{
WANG, X. M. - LI, X. D. - QIU, J. P. - LI, Y.S. ${ }^{*}$ \\ School of Agriculture and biology, Shanghai Jiao Tong University, Shanghai 200240, China \\ *Corresponding author \\ e-mail: yinshengli@sjtu.edu.cn \\ (Received $8^{\text {th }}$ Sep 2019; accepted $15^{\text {th }}$ Nov 2019)
}

\begin{abstract}
The multi-layer biofilter (MBF) is a promising tool for low-strength wastewater treatment. This paper investigates the microbial composition of a full-scale MBF system in the suburb of Shanghai, China, and analyzes the structure of the bacterial community with 454-pyrosequencing. The results show that different parts of the MBF, namely, the second, fourth and sixth layers (L2, L4 and L6) differed in bacteria composition. A total of 7,385, 7,042 and 5,974 sequences with high-quality $16 \mathrm{~S}$ rRNA genes were recovered from L2, L4 and L6, respectively, and subjected to community analysis. The libraries of L2, L4 and L6 consist of 1,457, 1,310 and 1,197 operational taxonomic units (OTUs), respectively. In addition, L2, L4 and L6, had 89, 87 and 80 classes of bacteria, respectively. However, 73 classes of L2, 70 classes of L4 and 62 classes of L6 had an abundance lower than 1\%. Besides, Thermus was only found in L2. This research is the first one ever to study the microbial communities in the biofilm of full-scale MBF systems in wastewater treatment. The research findings shed new light on the biological mechanism of contaminant removal in the MBF, and promote the application of the MBF in wastewater treatment.
\end{abstract}

Keywords: biofilter, 454 pyrosequencing, bacterial composition, community richness, wastewater

\section{Introduction}

Extended from conventional trickling filters, multi-layer biofilter (MBF) is a new wastewater treatment tool that effectively eliminates organic and nutrient contaminants, using a mixture of microorganisms (Ou et al., 2009).

Previously, the bacterial community in wastewater treatment plants has often been identified by culture-dependent methods. However, these methods can only cultivate and characterize less than $1 \%$ of the microorganisms in different habitats (Torsvik et al., 2002). In recent years, pyrosequencing has emerged as a popular method to examine the structure of bacterial communities. This approach can obtain varied genetic information on the microbes in the environment. For example, 454 Life Sciences (Branford, US) designed the 454 pyrosequencing, a high-throughput analytical method that utilizes a single strand of DNA with a length of 400 500bp. With the aid of 454 pyrosequencing, Wen et al. (2017) found that the population of ammonia oxidizing bacteria (AOB) shrank with the decline of ammonia concentration, but failed to observe a dramatic drop in the percentage of anammox bacteria. Lautenschlager (2014) applied 454 pyrosequencing to three biofilters with similar microbial composition, yet the results go against the actual situation. As an emerging microbial research method Roche 454-high throughput pyrosequecing have been used widely to analyze the microbial community in WWTPs (Ye et al., 2011; Zhang et al., 2012; Hu et al., 2012; Ye and Zhang, 2013; Pervin et al., 2013; Zheng et al., 2017; Liu et al., 2017).

Despite the above studies, there is little report that fully discloses the variation in microbial composition in wastewater treatment plants, especially in biofilters. Yang et al. 
(2013) compared vermifilter and the conventional biofilter, and drew the following conclusions: the biofilm of vermifilter has two types of bacterial strains (firmicutes and $\gamma$ - proteobacteria), while that of conventional biofilter has four types (firmicutes, $\gamma$ - proteobacteria, $\beta$-proteobacteria and actinobacteria).

This paper attempts to characterize and compare the bacteria community at different positions along the MBF. For this purpose, the author probed deep into the microbial composition of a full-scale MBF system in the suburb of Shanghai, China, and analyzed the structure of the bacterial community with 454-pyrosequencing.

\section{Materials and Methods}

\section{Research object}

Located in the suburb of Shanghai, China, our research object, i.e. a full-scale MBF system, has been operating stably for nearly 7 years (GPS, 31.587,121.656). The system consists of multiple modules of the same size $(\mathrm{L} \times \mathrm{W} \times \mathrm{H}: 675 \mathrm{~cm} \times 485 \mathrm{~cm} \times 400 \mathrm{~cm})$. A vertical gap of $15 \mathrm{~cm}$ is maintained between adjacent modules. The biofilm on the filler surface is in full contact with the air, allowing the microorganisms to get enough oxygen. From top to bottom, the biofilter chamber can be divided into seven layers. The upper four layers are filled with ceramists, and the lower three layers, volcanic rocks. The treatment efficiency is $500 \mathrm{~m}^{3}$ per day.

The technological process involves an equalization tank, a lifting pump, a MBF, an intermediate tank, and a constructed wetland. After being collected by the pipe network, the wastewater from the equalization tank is pumped by the lifting pump to the MBF, and be dispersed to all places through the water channel. From top to bottom, the sewage flows through $\mathrm{MBF}$, and then converges from the bottom to the sedimentation tank at the rear. and comes into full contact with the biofilm on the filler. The spilled part flows back to the middle pool. Then, the contaminants are absorbed and degraded by the microorganisms on the biofilm.

\section{Sample collection and processing}

The biofilm samples were collected from the second, fourth and sixth layers, and denoted as L2, L4 and L6, respectively. In order to ensure the reliability and uniformity of the samples, five-point sampling method is adopted, that is, sampling from five different positions of the same level, and mixing. Each sample was obtained from the filler by 5 min ultrasonic treatment, and then flushed with deionized water. Then, the sample was relocated to a $50 \mathrm{~mL}$ tube, and centrifuged at $8,000 \times \mathrm{g}$ for $10 \mathrm{~min}$. The sediments were stored at $-80^{\circ} \mathrm{C}$ until DNA extraction.

\section{DNA extraction and polymerase chain reactions (PCRs)}

The total genomic DNA was extracted by E.Z.N.A.® Soil DNA Kit (Omega Bio-tek, US). To construct the clone library, the primer set $341 \mathrm{~F}$ (5'-CCTACGGGAGGCAGCAG-3') and 907R (5'- CCGTCAATTCMTTTRAGT-3') was used to amplify the hypervariable V3 V5 region (about 560bp) of bacterial $16 \mathrm{~S}$ rRNA gene. The fused forward primer includes a10-base long barcode inserted between the 454 adapter and the $341 \mathrm{~F}$ primer, aiming to distinguish each sample in the mixed reaction. 
The PCRs were performed in a $25 \mu \mathrm{L}$ volume containing $2.5 \mu \mathrm{L} 10 \times$ buffer , $2 \mu \mathrm{L}$ $\mathrm{dNTP}, 1 \mu \mathrm{L}$ of each primer $(10 \mu \mathrm{M}), 2 \mu \mathrm{L}$ of DNA $(20 \mathrm{ng} / \mu \mathrm{L})$ and $0.125 \mu \mathrm{L}$ pyrobest polymerase $(5 \mathrm{U} / \mu \mathrm{L}$, Takara, China). The thermocycling was carried out in the following steps: $94^{\circ} \mathrm{C}$ for $4 \mathrm{~min}$, followed by 27 cycles at $95^{\circ} \mathrm{C}$ for $30 \mathrm{~s}, 55^{\circ} \mathrm{C}$ for $45 \mathrm{~s}, 72^{\circ} \mathrm{C}$ for $1 \mathrm{~min}$ and a final extension at $72^{\circ} \mathrm{C}$ for $7 \mathrm{~min}$. In this study, the number of PCR cycles is reduced to ensure the accuracy and reliability of the subsequent analysis. The PCR products were run on a $0.8 \%$ agarose gel, with the amplicon size of 500bp.

\section{Pyrosequencing}

The PCR products were purified using the AMPure XP beads, quantified by Quant-iT PicoGreen dsDNA Assay Kit (Thermo Fisher Scientific, US), and pyro-sequenced on 454 Gs Flx Titanium Platform (Roche, US) in Shanghai Personalbio Co., Ltd.

\section{Sequence analysis}

Following pyrosequencing, the genetic sequences were analyzed on QIIME, an opensource bioinformatics pipeline for performing microbiome analysis from raw DNA sequencing data. Firstly, the ineffective sequences were filtered out, leaving only sequences satisfying the following requirements: the length falls within 200 1,000bp, fewer than 6 consecutive bases are identical, no ambiguous base is included, and the quality score is above 25 .

The uchime_denovo command was called to remove chimera, which would otherwise affect sequence quality. Then, sequences with similarities of greater than $97 \%$ were clustered into one operational taxonomic unit (OTU), using the QIIME program. Community richness and diversity indices (i.e. Chao1 estimator, ACE estimator and Simpson's diversity) and rarefaction curves were obtained with mothur. The larger the Chao / ACE index is, the higher the community richness is. The larger the Simpson index is, the lower the community diversity is.

\section{Results and Discussion}

\section{MBF performance}

The water quality of MBF obtained by measuring three parallel water samples at different positions is as follows. The project has been in stable operation for several years, and the effluent quality has reached the national water quality discharge standard of China (GB 8978-1996).

Table 1 lists several water quality indices before and after the water treatment. During the treatment, the mean chemical oxygen demand (COD) of influent was $306 \mathrm{mg} / \mathrm{L}$, while the mean COD of effluent was 232, 215 and $227 \mathrm{mg} / \mathrm{L}$ for L2, L4 and L6, respectively. Thus, the COD removal rate of the MBF was $25.8 \%$.

The mean ammonia nitrogen $\left(\mathrm{NH}_{3}-\mathrm{N}\right)$ was $19 \mathrm{mg} / \mathrm{L}$ before the treatment. After the treatment, the mean $\mathrm{NH}_{3}-\mathrm{N}$ of effluent was $15.33,10.83$ and $9.54 \mathrm{mg} / \mathrm{L}$, for $\mathrm{L} 2, \mathrm{~L} 4$ and L6, respectively. Thus, the MBF removed $50 \%$ of the $\mathrm{NH}_{3}-\mathrm{N}$ from the wastewater.

The mean total nitrogen (TN) was $31.82 \mathrm{mg} / \mathrm{L}$ in the influent. After the treatment, the mean TN of effluent was 20.94, 29.48 and $27.76 \mathrm{mg} / \mathrm{L}$, for L2, L4 and L6, respectively. Therefore, the TN removal rate of the MBF was $12.8 \%$, lower than that of $\mathrm{NH}_{3}-\mathrm{N}$. 
The mean total phosphorus (TP) was $3.89 \mathrm{mg} / \mathrm{L}$ in the influent. After the treatment, the mean TP of effluent was $3.75,3.8$ and $3.7 \mathrm{mg} / \mathrm{L}$, for L2, L4 and L6, respectively. Hence, the MBF removed $4 \%$ of the TP from the influent.

Table 1. Water quality indices (temperature: $19.45 \pm 0.49^{\circ} \mathrm{C}$ )

\begin{tabular}{c|c|c|c|c|c|c}
\hline & $\mathbf{p H}$ & $\mathbf{D O}$ & $\mathbf{C O D}(\mathbf{m g} / \mathbf{L})$ & $\mathbf{N H}_{\mathbf{3}} \mathbf{- N}(\mathbf{m g} / \mathbf{L})$ & $\mathbf{T N}(\mathbf{m g} / \mathbf{L})$ & $\mathbf{T P}(\mathbf{m g} / \mathbf{L})$ \\
\hline Influent & 7.544 & 1.42 & 306 & 19 & 31.82 & 3.89 \\
L2 & 7.806 & 5.43 & 232 & 15.33 & 20.94 & 3.75 \\
L4 & 7.643 & 5.65 & 215 & 10.83 & 29.48 & 3.8 \\
L6 & 7.739 & 3.29 & 227 & 9.54 & 27.76 & 3.7 \\
\hline
\end{tabular}

\section{Bacteria diversity}

As shown in Table 2, a total of 7,385, 7,042 and 5,974 sequences with high-quality 16S rRNA genes were recovered from L2, L4 and L6, respectively, and subjected to community analysis. The libraries with L2, L4 and L6 consist of 1,457, 1,310 and 1,197 OTUs, respectively.

Table 2. Community richness and diversity indices

\begin{tabular}{c|c|c|c|c|c|c|c}
\hline $\begin{array}{c}\text { Sample } \\
\text { number }\end{array}$ & $\begin{array}{c}\text { Number of } \\
\text { effective } \\
\text { sequences }\end{array}$ & $\begin{array}{c}\text { Number of } \\
\text { high-quality } \\
\text { sequences }\end{array}$ & OTUs & $\begin{array}{c}\text { ACE } \\
\text { estimator }\end{array}$ & $\begin{array}{c}\text { Chao1 } \\
\text { estimator }\end{array}$ & $\begin{array}{c}\text { Simpson's } \\
\text { diversity }\end{array}$ & Coverage \\
\hline L2 & 11,239 & 7,385 & 1,457 & $3,164.202$ & $2,791.603$ & 0.002979 & 0.907651 \\
L4 & 10,840 & 7,042 & 1,310 & $2,905.24$ & $2,476.287$ & 0.004794 & 0.912099 \\
L6 & 9,611 & 5,974 & 1,197 & $2,446.65$ & $2,164.788$ & 0.004775 & 0.907934 \\
\hline
\end{tabular}

The rarefaction curves were plotted (Figure 1) based on the OTUs at 3\% cutoff to compare the community richness of the three samples. As shown in Figure 1, none of the curves approached a plateau, suggesting that further sequencing will continue to increase the number of OTUs in each sample. However, the coverage of all three samples had already reached $90 \%$. Pyrosequencing analysis output numerous OTUs, providing the genetic information needed to find scarce species (Zhang, 2011).

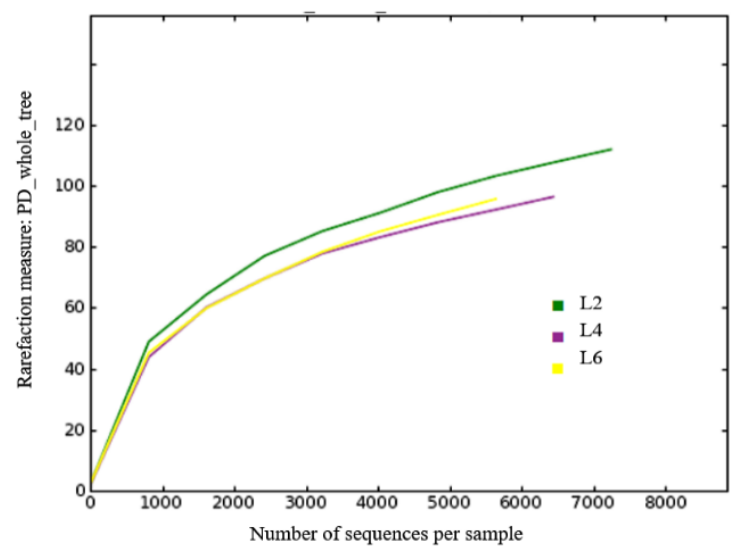

Figure 1. Rarefaction curves of OTUs in L2, L4 and L6 
It can also be seen from Table 2 that $\mathrm{L} 2$ contained richer species than any other sample. Besides, the values of ACE estimator, Chaol estimator, and Simpson's diversity show that L2 had the highest bacterial diversity, followed by L4 and then L6. The ranking of bacterial diversity agrees with the positions of the three layers in the MBF: the wastewater first flows through L2 first, then through L4, and finally through L6. In other words, the bacteria diversity decreases along the MBF. This conclusion echoes with the number of OTUs in these layers.

Next, the samples were sorted in descending order of OTU abundance, and the distribution curves of OTU abundance were plotted (Figure 2), taking $\log 2$ of abundance. In Figure 2, the horizontal length of each curve reflects the community richness of the corresponding sample, while the curve shape demonstrates species evenness. Obviously, the three samples were ranked as L2>L4>L6 in abundance, that is, the abundance also decreased along the MBF. It means that the more abundant the species are, the higher the evenness is reflected by the curve shape. By contrast, there was no significant difference among the samples in species evenness.

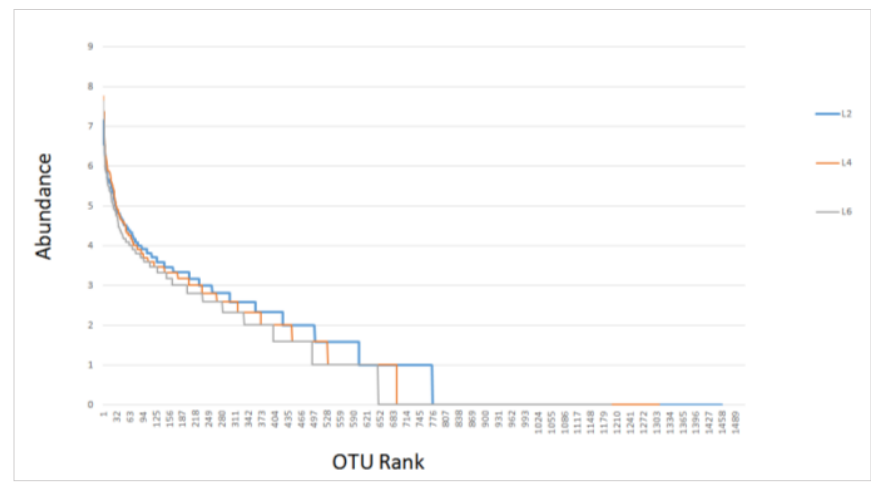

Figure 2. Distribution curves of OTU abundance

The Venn diagram of OTUs (Figure 3) was drawn by Venny 2.1.0 (http://bioinfogp.cnb.csic.es/tools/venny/index.html). An evident difference could be observed along the MBF at the genus-level. The difference between L4 and L6 was particularly obvious, revealing the function division in the filter. This result is confirmed by the subsequent analysis on bacterial composition.

\section{Bacteria composition}

The total number of phyla identified in L2, L4 and L6 was 34, 32 and 29, respectively, which, as previously discovered, decreases along the MBF. Here, a phylum with no less than $0.5 \%$ relative abundance is called a major phylum. Figure 4 shows the proportion of sequences in each phylum in the total number of sequences in each sample. The sequences that do not belong to any known phylum were categorized as unclassified bacteria.

As shown in Figure 5, L2 mainly contains proteobacteria (54.18\%), Bacteroidetes $(14.07 \%)$, actinobacteria $(9.13 \%)$ and nitrospirae $(5.06 \%)$. The bacteria in L4 mostly belong to proteobacteria (52.47\%), acidobacteria (15.04\%) and bacteroidetes $(11.87 \%)$. The major bacterial groups in L6 include proteobacteria $(54.18 \%)$, acidobacteria $(12.45 \%)$, bacteroidetes $(12.29 \%)$ and nitrospirae $(6.58 \%)$. To sum up, the three samples differed slightly in major bacterial groups. 
According to the comparison between the vermifilter and the conventional biofilter (Yang, 2013), the bacteria composition of the MBF is distinct from that of these two filters.

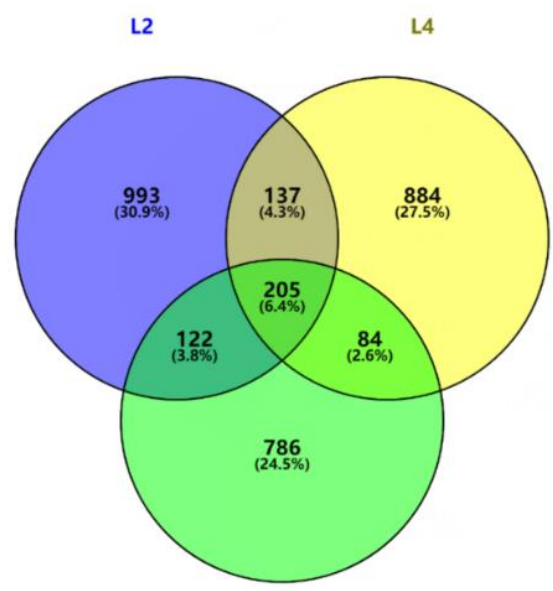

L6

Figure 3. The Venn diagram of OTUs

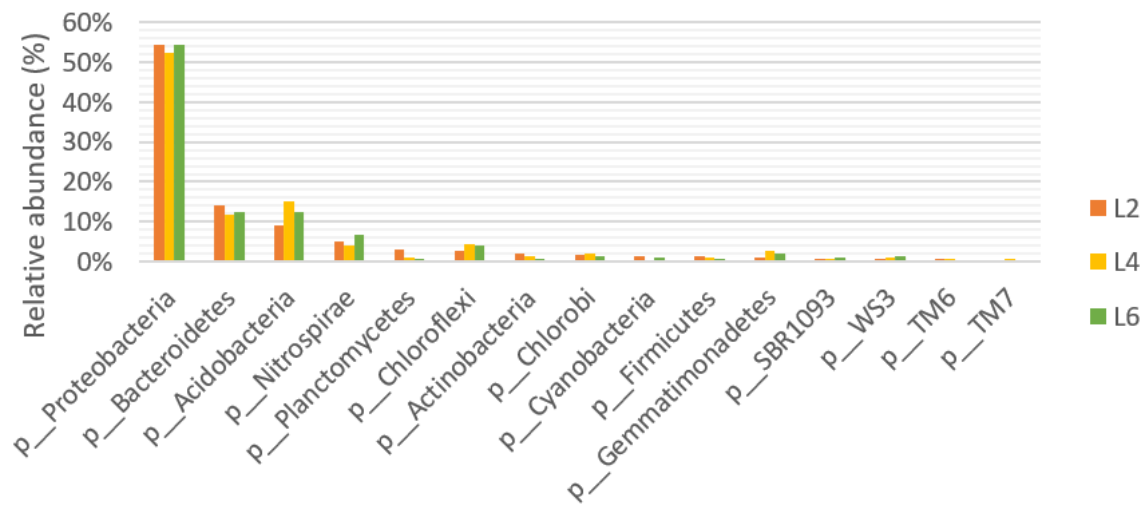

Figure 4. Proportion of sequences in each phylum

However, the three samples had marked differences in the class level of taxonomic classifications. As shown in Table 3, $\alpha$-proteobacteria, $\beta$-proteobacteria, $\delta$-proteobacteria and $\gamma$-proteobacteria and TA18 were found in all three samples, but the content of each component varied greatly from sample to sample. Along the MBF, the content of $\alpha-$ proteobacteria increased first and then decreased; the content of $\beta$-proteobacteria declined all the way; the contents of $\gamma$-proteobacteria and $\delta$-proteobacteria reduced first and then rebounded. Meanwhile, zetaproteobacteria, exclusively belonging to L6, was rare and represented by a single described species, Mariprofundus ferrooxydans. It is an ironoxidizing neutrophilic chemolithoautotroph first discovered in 1996, at the hydrothermal vents at Loihi Seamount, Hawaii (Emerson, 2002, 2007). Then, the dominant classes were $\delta$-proteobacteria, clostridia and $\gamma$-proteobacteria. 
The differences in bacteria content are attributable to various factors, such as climate zone, wastewater composition and weather condition. Most bacteria in the biofilter are not involved in the key processes of the wastewater treatment.

In total, a total of 89, 87 and 80 classes of bacteria were identified for L2, L4 and L6, respectively. However, 73 classes of L2, 70 classes of L4 and 62 classes of L6 had an abundance less than $1 \%$. In addition, Thermus was only found in L2.

The research of MBF mainly focuses on the improvement of structure and the selection of filler (Cui, 2014), but there is no research on microorganism.

Most of the biofilters with stratification, such as tower biofilters, are mostly anaerobic, and most of the microorganisms are anaerobic, which is far from the MBF in this paper (Malakar, 2017).

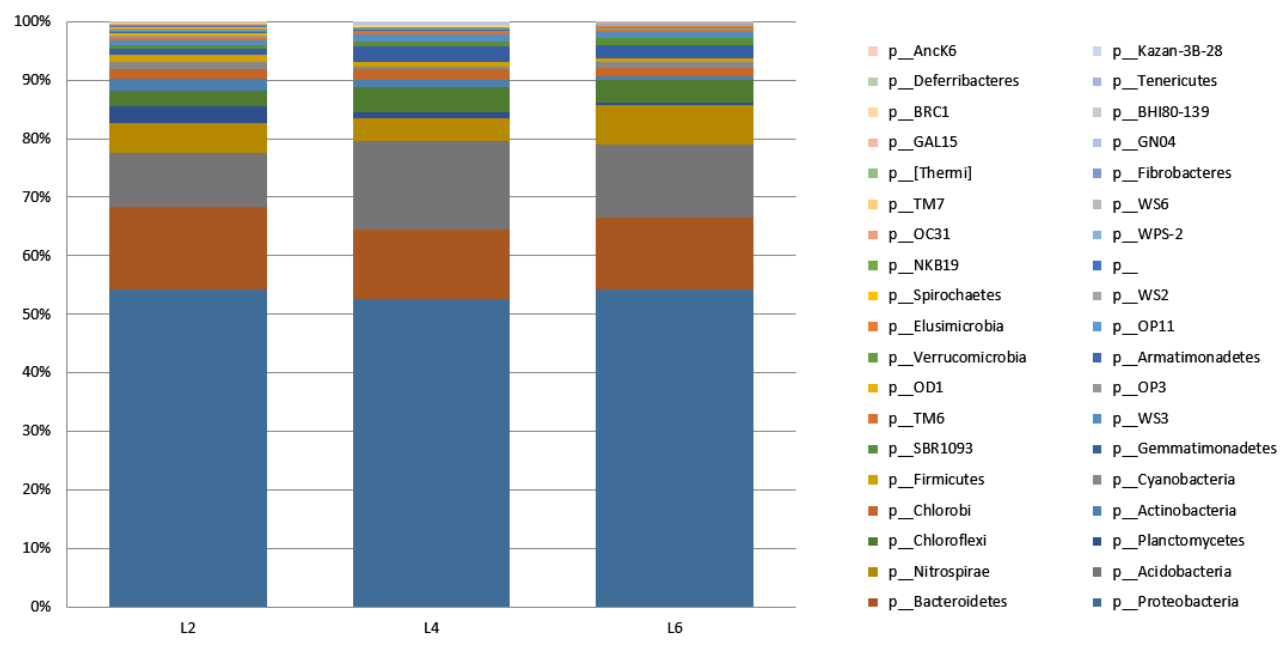

Figure 5. The proportions of sequences in different classes

Table 3. The class level of proteobacteria

\begin{tabular}{c|c|c|c|c|c|c}
\hline $\mathbf{L 2}$ & $\mathbf{( \% )}$ & $\mathbf{L 4}$ & $\mathbf{( \% )}$ & $\mathbf{L 6}$ & $\mathbf{( \% )}$ & \\
\hline 1,112 & $27.79 \%$ & 1436 & $38.86 \%$ & 988 & $30.52 \%$ & $\alpha$-proteobacteria \\
1,063 & $26.57 \%$ & 866 & $23.44 \%$ & 1,015 & $31.36 \%$ & $\gamma$-proteobacteria \\
954 & $23.84 \%$ & 890 & $24.09 \%$ & 656 & $20.27 \%$ & $\beta$-proteobacteria \\
833 & $20.82 \%$ & 461 & $12.48 \%$ & 563 & $17.39 \%$ & $\delta$-proteobacteria \\
29 & $0.72 \%$ & 19 & $0.51 \%$ & 12 & $0.37 \%$ & TA18 \\
10 & $0.25 \%$ & 23 & $0.62 \%$ & 2 & $0.06 \%$ & No blast hit \\
0 & $0.00 \%$ & 0 & $0.00 \%$ & 1 & $0.03 \%$ & Zetaproteobacteria \\
\hline
\end{tabular}

\section{Conclusions}

To our knowledge, this is the first time to investigate microbial communities in the biofilm of the full-scale MBF system for wastewater treatment. Through the analysis on MBF performance, bacteria diversity and bacteria composition, it is concluded that different parts of the MBF system vary greatly in bacteria composition. The bacteria of L2, L4 and L6 belong to 89, 87 and 80 classes, respectively. However, 73 classes of L2, 70 classes of L4 and 62 classes of L6 had an abundance less than 1\%. In addition, 
Thermus was only found in L2. The research findings provide new insights into the biological mechanism of contaminant removal in the MBF system.

Suggestions for future improvement are as follows. In engineering, the ventilation performance is increased while the aesthetics is ensured, for example, more louvers are used. Microorganisms need certain temperature conditions. It is suggested that heat preservation and dissipation should be considered when using this process in other places. Improving the efficiency of biological treatment can increase the porosity and specific surface area of the material. The particle size of the material has a certain influence on the good sample condition and sludge removal, which can be further studied. The roughness of the material surface also has a positive effect on the formation of biofilm. If the cost allows, increasing the number of layers within a certain range can improve the diversity of microorganisms. The nitrification of the lower layer is stronger than that of the upper layer. It is suggested that the materials should also be studied from the environment suitable for the growth of nitrifying bacteria.

\section{REFERENCES}

[1] Cui, T. T., He, X. J., Ling, R., Sun, X., Li, X. D. (2014): Nitrogen and phosphorus removal performance in the novel multi-layered biological tricking filter. - Journal of Agricultural Resources \& Environment.

[2] Emerson, D., Moyer, C. L. (2002): Neutrophilic fe-oxidizing bacteria are abundant at the loihi seamount hydrothermal vents and play a major role in Fe oxide deposition. - Applied and Environmental Microbiology 68(6): 3085-3093.

[3] Emerson, D., Rentz, J. A., Lilburn, T. G., Davis, R. E., Aldrich, H., Chan, C., Moyer, C. L. (2007): A novel lineage of proteobacteria involved in formation of marine fe-oxidizing microbial mat communities. - PLOS ONE 2.

[4] Hu, M., Wang, X., Wen, X., Xia, Y. (2012): Microbial community structures in different wastewater treatment plants as revealed by 454-pyrosequencing analysis. - Bioresour Technol 117: 72-79.

[5] Lautenschlager, K., Hwang, C., Ling, F., Liu, W. T., Boon, N., KoSter, O., Egli, T., Hammes, F. (2014): Abundance and composition of indigenous bacterial communities in a multi-step biofiltration-based drinking water treatment plant. - Water Research 62(Complete): 40-52.

[6] Liu, T., Mao, Y. J., Shi, Y. P., Quan, X. (2017): Start-up and bacterial community compositions of partial nitrification in moving bed biofilm reactor. - Appl Microbiol Biotechnol 101(6): 2563-2574.

[7] Malakar, S., Saha, P. D., Baskaran, D., Rajamanickam, R. (2017): Microbial biofilter for toluene removal: performance evaluation, transient operation and theoretical prediction of elimination capacity. - Sustainable Environment Research 28(3): 121-127.

[8] Ou, W. T., Li, X. D., Pang, H. R., Dai, Y. Q., Qiu, J. P. (2009): Treatment of Rural Sewage by Using Combined Processes of Multi-Layered Biological Filter and Constructed Wetland. - Water Purification Technology.

[9] Torsvik, V., Ovreas, L. (2002): Microbial diversity and function in soil: from genes to ecosystems. - Current Opinion in Microbiology 5(3): 240-245.

[10] Wen, X., Gong, B., Zhou, J., He, Q., Qing, X. (2017): Efficient simultaneous partial nitrification, anammox and denitrification (snad) system equipped with a real-time dissolved oxygen (do) intelligent control system and microbial community shifts of different substrate concentrations. - Water Research 119: 201-211.

[11] Yang, J., Liu, J., Xing, M., Lu, Z., Yan, Q. (2013): Effect of earthworms on the biochemical characterization of biofilms in vermifiltration treatment of excess sludge. - Bioresource Technology 143(Complete): 10-17. 
[12] Ye, L., Shao, M. F., Zhang, T., Tong, A. H., Lok, S. (2011): Analysis of the bacterial community in a laboratory-scale nitrification reactor and a wastewater treatment plant by 454-pyrosequencing. - Water Res 45(15): 4390-4398.

[13] Ye, L., Zhang, T. (2013): Bacterial communities in different sections of a municipal wastewater treatment plant revealed by 16S rDNA 454 pyrosequencing. - Appl Microbiol Biotechnol 97(6): 2681-2690.

[14] Zhang, X., Yue, S., Zhong, H., Hua, W., Chen, R., Cao, Y. (2011): A diverse bacterial community in an anoxic quinoline-degrading bioreactor determined by using pyrosequencing and clone library analysis. - Applied Microbiology \& Biotechnology 91(2): 425-434.

[15] Zhang, T., Shao, M. F., Ye, L. (2012): 454 pyrosequencing reveals bacterial diversity of activated sludge from 14 sewage treatment plants. - ISME J 6(6): 1137-1147.

[16] Zheng, M., Zuo, Z. Q., Zhang, Y. Z., Cui, Y. J., Dong, Q., Liu, Y. C. (2017): Nitrite production from urine for sulfide control in sewers. - Water Res 122: 447-454. 